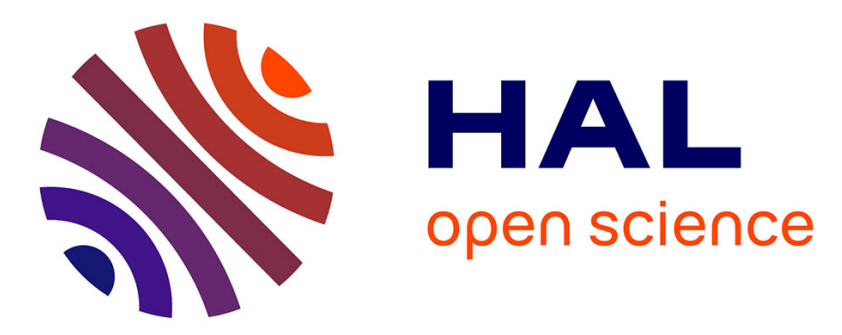

\title{
Effet de divers amendements organiques sur les rendements du maïs et de la pomme de terre cultivés en sol sableux
}

J. Delas, C. Molot

\section{To cite this version:}

J. Delas, C. Molot. Effet de divers amendements organiques sur les rendements du maïs et de la pomme de terre cultivés en sol sableux. Agronomie, 1983, 3 (1), pp.19-26. hal-02722871

\section{HAL Id: hal-02722871 \\ https://hal.inrae.fr/hal-02722871}

Submitted on 1 Jun 2020

HAL is a multi-disciplinary open access archive for the deposit and dissemination of scientific research documents, whether they are published or not. The documents may come from teaching and research institutions in France or abroad, or from public or private research centers.
L'archive ouverte pluridisciplinaire HAL, est destinée au dépôt et à la diffusion de documents scientifiques de niveau recherche, publiés ou non, émanant des établissements d'enseignement et de recherche français ou étrangers, des laboratoires publics ou privés. 


\title{
Effet de divers amendements organiques sur les rendements du maïs et de la pomme de terre cultivés en sol sableux
}

\author{
Jacques DELAS \& Christian MOLOT \\ I.N.R.A., Station d'Agronomie, Centre de Recherches de Bordeaux, F 33140 Pont-de-la-Maye
}

\author{
RÉSUMÉ \\ Amendement organique, \\ Paille, \\ Ecorces, \\ Marcs de raisin, \\ Fumier, \\ Tiges de maïs, \\ Matière organique, \\ Maïs, \\ Pomme de terre, \\ Sols sableux, \\ Fertilité des sols.
}

\begin{abstract}
Nous avons étudié pendant 12 ans, dans les conditions naturelles, l'effet de l'épandage annuel d'amendements organiques sur les rendements du maïs et de la pomme de terre cultivés dans un sol sableux irrigué. Les diverses matières organiques (marcs de raisin « $R$ », écorces de résineux « $E$ », tiges de maïs « $M$ », fumier de ferme « $F$ », paille de blé « $P$ ») contenaient la même quantité de carbone ; des compensations minćralcs ont permis de réaliser en outre l'égalité des apports de $\mathrm{N}, \mathrm{P}, \mathrm{K}, \mathrm{Ca}$ et $\mathrm{Mg}$ entre traitements (y compris le témoin « $T »)$ et d'assurer un niveau de fertilisation minérale élevé.

Dans ces conditions, les amendements ont tous provoqué des augmentations de rendement par rapport au témoin, significatives 8 années sur 12 ; l'effet des différents amendements a été identique, malgré des taux d'enrichissement du sol en matière organique très différents.

L'origine de l'efficacité des amendements n'a pas été clairement établie ; il est possible cepcndant qu'une amélioration de l'alimentation hydrique et minérale des cultures, provoquée par l'augmentation de la capacité de rétention pour l'eau et de la capacité d'échange de cations d'un sol très filtrant, ait joué un rôle important. Ces résultats confirment le rôle essentiel de la matière organique dans la fertilité des sols sableux et, donc, la nécessité d'en maintenir le taux à un niveau suffisant par la restitution des résidus de culture ou par l'apport d'amendements. Nous avons pu calculer le rendement en carbone stable des diverses sources utilisées et mettre en évidence la valeur de produits encore peu connus (marcs de raisin, écorces de résineux) pour l'amélioration de l'état organique des sols.
\end{abstract}

\section{SUMMARY}

Organic materials,

Straw,

Bark,

Grape marc,

Farmyard manure,

Maize stalks,

Organic matter,

Maize,

Potatoes,

Sandy soils,

Soil fertility.

\section{Effect of various organic materials on the yield of maize and potatoes on sandy soil}

For 12 years, we have studied under natural conditions the effect of the annual addition of organic materials on the yield of maize and potatoes cropped in an irrigated sandy soil. The various organic additives (grape marc « $R$ », barks « $E$ », maize stalks « $M$ », farmyard manure « $F$, wheat straw « $P$ ») contained the same amount of carbon; further mineral adjustments were made to equalize the rates of $\mathrm{N}, \mathrm{P}, \mathrm{K}, \mathrm{Ca}$ and $\mathrm{Mg}$ in all the treatments (including the control « $T »)$ and to maintain a high level of mineral fertilization.

Under these conditions, and for 8 years out of 12 , all the organic additives significantly improved yield in relation to the control. The effect of the various additives was identical despite a quite variable effect on the level of enrichment of the soil in organic matter.

The reasons for the efficiency of these additives have not been clearly established : however it may be that the improvement of the water and mineral availability to the crop, caused by the increase in water retention and cation exchange capacities of a very porous soil, play an important role.

These results confirm the essential role of organic matter in the fertility of sandy soils and the absolute requirement to maintain its content at a certain level, by incorporating crop residues or by adding organic additives. We have calculated the yield in stable carbon of the various additives used and demonstrated the value of little known products such as grape marc, softwood bark, etc. for improving the organic status of the soil.

\section{INTRODUCTION}

En conclusion d'une mise au point sur les effets des matières organiques, nous avons remarqué que l'action sur les rendements des fumures organiques et des fumures strictements minérales, dans la mesure où elles apportent au sol des quantités d'éléments minéraux identiques, est la même (DElas et al., 1973). Nous avons attribué la contradiction entre cette absence d'efficacité des matières organiques et l'effet considérable qu'elles exercent sur les propriétés des sols au fait que les études avaient été conduites dans des sols bien pourvus en matière organique, ou bien dans des sols où l'évolution de cette dernière était ralentie par la présence de calcaire. 
Les sols sableux, pauvres en humus et colloïdes minéraux, acides, devraient se prêter beaucoup mieux aux recherches concernant l'intérêt éventuel des amendements organiques : de tels sols sont nombreux dans le vignoble bordelais et, depuis l'abandon des restitutions traditionnelles, ils s'appauvrissent en humus au rythme de 2 à 3 p. 100 par an (DELAS, 1968).

C'est la raison pour laquelle nous avons entrepris, il y a une vingtaine d'années, des études basées sur des expérimentations en plein champ, concernant l'entretien humique des sols viticoles du Bordelais et les rôles de la matière organique dans ces sols. Certains essais, établis dans les conditions de la pratique agricole, sont consacrés à l'étude de l'effet de divers amendements organiques sur le comportement de la vigne et les propriétés des sols viticoles. Mais le terme de telles études concernant une plante pérenne est éloigné ; pour obtenir des réponses plus rapides permettant d'orienter une politique d'entretien humique des sols viticoles, nous avons mis en place d'autres essais en microparcelles ne portant pas de cultures ou bien des cultures annuelles.

Les résultats d'une première expérimentation de 7 ans, concernant l'action de différentes matières organiques sur les propriétés d'un sol sableux non cultivé, ont été publiés (DELAS, 1971). Nous avons montré que les propriétés les plus liées à la teneur en matière organique étaient la capacité d'échange de cations, la capacité de rétention pour l'eau, l'activité biologique, la teneur en éléments fertilisants.

Dans cet article, nous exposerons les résultats obtenus après 12 ans sur un essai établi dans le même type de sol et destiné à mesurer l'effet d'amendements variés sur les rendements de deux cultures. Nous essaierons, dans la discussion, d'aller au-delà des considérations viticoles qui sont à l'origine de ce travail et de situer nos résultats dans le cadre plus général des préoccupations sur l'évolution de la fertilité des sols ou la valorisation des déchets organiques.

\section{MATÉRIEL ET MÉTHODES}

\section{A. Protocole expérimental}

L'essai a été implanté au domaine I.N.R.A. de la Grande-Ferrade, dans une parcelle en très faible pente ayant porté précédemment de la vigne puis des arbres fruitiers. Le climat est du type océanique tempéré humide. Le sol, qui appartient à la terrasse moyenne de la Garonne (étage 1b), est du type brun légèrement lessivé ; ses caractéristiques initiales moyennes sont indiquées au tableau 1. C'est un «sol de Graves» (40 p. 100 de cailloux et graviers), de texture sableuse, très pauvre en matière organique ; la valeur élevée du pH est la conséquence d'apports importants de scories effectués quelques années avant la mise en place de l'essai.

Le dispositif expérimental comporte 48 parcelles réparties en 4 blocs. Le nombre de traitements (répétés 4 fois) a été de 12 jusqu'en 1970:6 types d'amendements et culture ou non culture des parcelles, les parcelles laissées nues étant destinées à l'étude de l'évolution de l'azote minéral. En 1971, l'ensemble de l'essai a été mis en culture et le dispositif n'a plus comporté que 6 traitements (répétés 8 fois). Cinq amendements, apportés annuellement, sont comparés à un témoin « $T$ » ne recevant qu'une fumure strictement minérale: marcs de raisin « $\mathbf{R}$ », écorces de

\section{TABLEAU 1}

Caractéristiques initiales moyennes du sol de l'essai $(0-20 \mathrm{~cm})$. Initial mean characteristics of the experimental soil $(0-20 \mathrm{~cm})$.

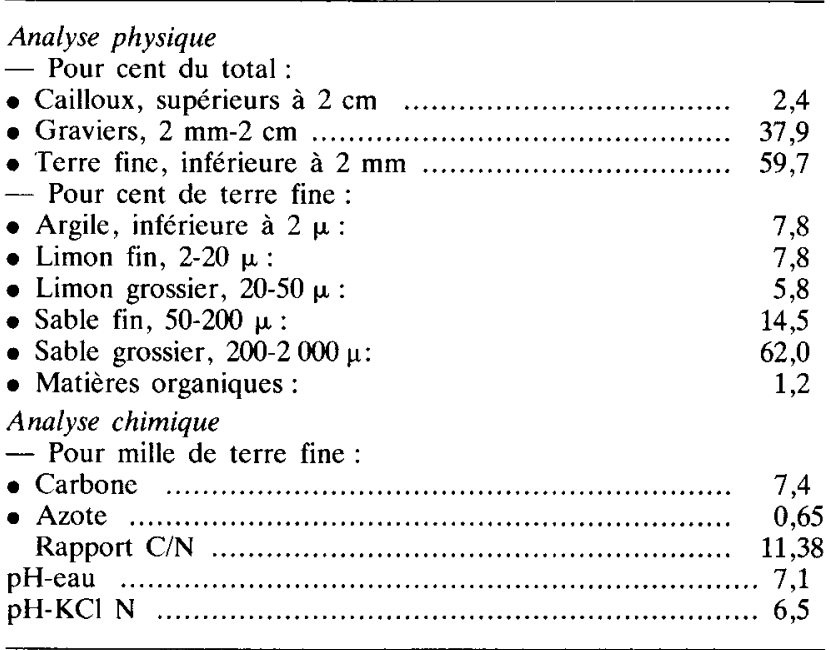

résineux « $E$ » $\left({ }^{1}\right)$, tiges de maïs « $M$ », fumier de ferme «F », paille de blé « $\mathbf{P} »$. La dose d'épandage est calculée de telle sorte qu'un même apport de carbone soit réalisé par tous les amendements et pour toutes les années. La dose de carbone choisie (environ $3 \mathrm{t} / \mathrm{ha} / \mathrm{an}$ ) correspond à l'apport annuel de $35 \mathrm{t} / \mathrm{ha}$ de fumier. La composition moyenne des matières organiques utilisées, les doses moyennes d'emploi ainsi que les quantités totales d'éléments minéraux apportées par ces matières organiques depuis le début de l'essai sont indiquées dans le tableau 2.

En outre, des compensations minérales permettent de réaliser chaque année l'égalité des apports de $\mathrm{N}, \mathrm{P}, \mathrm{K}, \mathrm{Ca}$, $\mathrm{Mg}$ dans les différents traitements; les complémentations nécessaires sont effectuées (y compris dans le témoin) en prenant pour référence, pour chaque élément, la quantité contenue dans l'amendement le plus riche. Nous cherchons par ce moyen à éviter d'attribuer à un effet spécifique de la matière organique un effet de la teneur en éléments minéraux de l'amendement.

Une autre compensation est réalisée chaque année, afin de maintenir constant le niveau initial de fertilité du sol : les quantités de N, P, K, Ca et Mg exportées des parcelles par les récoltes (mesurées par l'analyse) sont réincorporées au sol. Toutes les compensations en calcium et en magnésium sont effectuées à l'aide de sulfates.

Nous avons récapitulé dans le tableau 3 l'ensemble des apports réalisés en 12 ans dans les différents traitements (en nous limitant à $\mathrm{N}, \mathrm{P}$ et $\mathrm{K}$ ). L'examen de ce tableau fait apparaitre que, si le principe de la double compensation conduit à des apports assez variables selon les traitements, les quantités épandues sont du même ordre de grandeur et importantes.

Les parcelles élémentaires sont de $9 \mathrm{~m}^{2}$; elles sont séparées les unes des autres par une allée de $0,50 \mathrm{~m}$; les blocs sont limités par une allée de $1 \mathrm{~m}$.

(') Les apports d'écorces n'ont commencé qu'en 1970, après 3 années d'apports de foin de luzerne. Nos résultats précédents ayant montré que le foin de luzerne changeait peu les propriétés d'un sol sableux (DELAs, 1971), nous avons estimé pouvoir modifier en cours d'essai le protocole initial pour répondre à des préoccupations nouvelles concernant la valorisation des écorces. 
TABLEAU 2

Caractéristiques des apports de matières organiques effectués de 1967 à 1978.

Selected chemical properties of organic manures supplied from 1967 to 1978.

\begin{tabular}{|c|c|c|c|c|c|c|c|c|c|c|c|c|c|c|c|c|}
\hline \multirow[t]{2}{*}{ Traitements } & \multicolumn{2}{|c|}{$\begin{array}{c}\text { Poids frais } \\
\text { des matières } \\
\text { organiques } \\
\text { épandues }(\mathrm{kg} / \mathrm{ha})\end{array}$} & \multicolumn{8}{|c|}{$\begin{array}{c}\text { Composition moyenne des matières organiques épandues } \\
\text { en pour cent de la matière sèche }\end{array}$} & \multicolumn{6}{|c|}{$\begin{array}{l}\text { Quantités totales d’éléments } \\
\text { apportés par les matières organiques } \\
\text { de } 1967 \text { à } 1978(\mathrm{~kg} / \mathrm{ha})\end{array}$} \\
\hline & Total & $\begin{array}{c}\text { Par an } \\
\text { (moyenne) }\end{array}$ & $\%$ & $\mathrm{C}$ & $\mathrm{N}$ & $\mathrm{C} / \mathrm{N}$ & $\mathbf{P}$ & K & $\mathrm{Ca}$ & $\mathrm{Mg}$ & $\mathrm{C}$ & $\mathrm{N}$ & $P$ & K & $\mathrm{Ca}$ & $\mathrm{Mg}$ \\
\hline $\mathrm{T}$ Témoin & 0 & 0 & & & & & & & & & 0 & 0 & 0 & 0 & 0 & 0 \\
\hline $\mathrm{R}$ Marcs de raisin & 286121 & 23843 & 23,30 & 47,50 & 2,25 & 21,11 & 0,24 & 1,89 & 0,85 & 0,09 & 31666 & 1500 & 160 & 1260 & 567 & 60 \\
\hline E Ecorces de résineux* & 122861 & 10238 & 54,09 & 47,65 & 1,10 & 52,42 & 0,09 & 0,67 & 0,95 & 0,08 & 31666 & 731 & 60 & 445 & 631 & 53 \\
\hline M Tiges de mais & 261500 & 21791 & 28,40 & 45,48 & 0,85 & 53,51 & 0,12 & 1,57 & 0,38 & 0,12 & 33776 & 631 & 89 & 1166 & 282 & 89 \\
\hline F Fumier de ferme & 438392 & 36532 & 21,45 & 33,68 & 1,92 & 17,54 & 0,43 & 1,81 & 1,73 & 0,35 & 31671 & 1805 & 404 & 1702 & 1627 & 329 \\
\hline P Paille de blé & 113338 & 9444 & 63,14 & 44,25 & 0,41 & 107,93 & 0,06 & 0,85 & 0,31 & 0,08 & 31667 & 293 & 43 & 608 & 222 & 57 \\
\hline
\end{tabular}

* Ces données correspondent aux apports cumulés de foin de luzerne (1967 à 1969) et d'écorces de résineux (1970 à 1978); les chiffres relatifs à chacun des deux amendements sont indiqués ci-dessous :

$\begin{array}{lcrrrrrrrrrrrrrrr}\text { Foin de luzerne } & 47945 & 15982 & 58,14 & 45,44 & 2,33 & 19,50 & 0,20 & 1,47 & 1,77 & 0,14 & 12666 & 649 & 55 & 410 & 494 & 39 \\ \text { Ecorces de résineux } & 74916 & 8324 & 51,51 & 49,24 & 0,22 & 223,82 & 0,02 & 0,09 & 0,35 & 0,04 & 19000 & 84 & 7 & 35 & 135 & 15\end{array}$

\section{TABLEAU 3}

Quantités (en $\mathrm{kg} / \mathrm{ha}$ ) de N, P et $\mathrm{K}$ apportées entre 1967 et 1978 dans les traitements.

Total N, P and K amounts supplied in plots from 1967 to $1978(\mathrm{~kg} / \mathrm{ha})$.

\begin{tabular}{|c|c|c|c|c|c|c|c|c|c|c|c|c|c|c|c|}
\hline & \multicolumn{15}{|c|}{ Eléments apportés par les fumures minérales: } \\
\hline & \multicolumn{3}{|c|}{$\begin{array}{c}\text { Eléments apportés } \\
\text { par les matières } \\
\text { organiques }\end{array}$} & \multicolumn{3}{|c|}{$\begin{array}{l}\text { Pour réaliser } \\
\text { l'égalité des apports } \\
\text { entre matières } \\
\text { organiques }\end{array}$} & \multicolumn{3}{|c|}{$\begin{array}{l}\text { Pour compenser } \\
\text { les exonérations } \\
\text { par les récoltes }\end{array}$} & \multicolumn{3}{|c|}{ Total } & \multicolumn{3}{|c|}{$\begin{array}{c}\text { Total } \\
\text { Général }\end{array}$} \\
\hline & $\mathbf{N}$ & $\mathrm{P}$ & $\mathbf{K}$ & $\mathbf{N}$ & $\mathbf{P}$ & $\mathbf{K}$ & $\mathbf{N}$ & $\mathbf{P}$ & $\mathrm{K}$ & $\mathbf{N}$ & $\mathbf{P}$ & $\mathrm{K}$ & $\mathbf{N}$ & $\mathbf{P}$ & $\mathbf{K}$ \\
\hline T Témoin & 0 & 0 & 0 & 2001 & 404 & 2085 & 1631 & 230 & 1660 & 3632 & 634 & 3745 & 3632 & 634 & 3745 \\
\hline $\mathrm{R}$ Marcs de raisin & 1500 & 160 & 1260 & 501 & 244 & 825 & 1874 & 309 & 2380 & 2375 & 553 & 3205 & 3875 & 713 & 4465 \\
\hline E Ecorces de résineux & 731 & 60 & 445 & 1270 & 344 & 1640 & 2099 & 294 & 2382 & 3369 & 638 & 4022 & 4100 & 698 & 4567 \\
\hline M Tiges de maïs & 631 & 89 & 1166 & 1370 & 315 & 919 & 2104 & 336 & 2476 & 3474 & 651 & 3395 & 4105 & 740 & 4561 \\
\hline F Fumier de ferme & 1805 & 404 & 1702 & 196 & 0 & 383 & 1800 & 295 & 2186 & 1996 & 295 & 2569 & 3801 & 699 & 4271 \\
\hline P Paille de blé & 293 & 43 & 608 & 1708 & 361 & 1477 & 1927 & 288 & 2236 & 3635 & 649 & 3713 & 3928 & 692 & 4321 \\
\hline
\end{tabular}

Les matières organiques sont enfouies à la bêche dans les 20 premiers $\mathrm{cm}$ de sol, en décembre ou janvier de chaque année. Les compléments minéraux sont apportés à la mise en place des cultures pour la totalité de $\mathrm{P}, \mathrm{K}, \mathrm{Ca}, \mathrm{Mg}$ et pour la moitié de l'azote : le reste de l'azote est épandu en cours de culture, un mois après.

Les résidus de récolte (parties aériennes du maïs et de la pomme de terre) ne sont pas réincorporés au sol.

Deux types de culture ont été réalisés :

- en 1967, 1968, 1969 et 1977 : maïs grain (irrigué),

— de 1970 à 1976 et en 1978: pommes de terre (irriguées).

Les déterminations concernent le poids frais et sec des récoltes (grains et tiges de mais, tubercules et fanes de pomme de terre) et leur teneur en éléments minéraux ( $N, P$, $\mathrm{K}, \mathrm{Ca}, \mathrm{Mg}$ ), le nombre de tubercules de pomme de terre. Des analyses chimiques du sol de toutes les parcelles ont été réalisées avant la mise en place de l'essai et après la dernière récolte.

\section{B. Méthodes analytiques utilisées}

Les analyses ont été effectuées par les laboratoires d'analyses de l'I.N.R.A. d'Arras (sols) et de Pont-de-laMaye (sols et végétaux) qui ont utilisé les méthodes suivantes :

- Sols

- $\mathrm{N}$ total : méthode Kjeldahl,

- C organique : méthode Anne (1945),

- $\mathrm{P}_{2} \mathrm{O}_{5}$ assimilable : extraction par l'acide citrique à 2 p. 100 (méthode Dyer) puis dosage de $\mathrm{P}$ par colorimétrie du complexe phosphomolybdique réduit.

- $\mathrm{K}, \mathrm{Mg}$, Ca échangeables : extraction par l'acétate d'ammonium $\mathrm{N}$ à $\mathrm{pH} 7$ et dosage par photométrie de flamme ou d'absorption atomique. 


\section{- Végétaux}

- $\mathrm{N}$ : méthode Kjeldahl,

- $\mathrm{P}, \mathrm{K}, \mathrm{Ca}, \mathrm{Mg}$ : minéralisation par voie sèche puis : P: colorimétrie du complexe phosphomolybdique réduit,

$\mathrm{K}, \mathrm{Ca}, \mathrm{Mg}$ : photométrie de flamme ou d'absorption atomique.

\section{RÉSULTATS}

\section{A. Effet des matières organiques sur les rendements}

\section{Maïs (tabl. 4)}

Le maïs a été cultivé pendant 4 années $(1967,1968,1969$ et 1977). Les 3 premières années de l'essai, il n'y a aucune différence significative entre les traitements (répétés 4 fois). En 1977, par contre, les 5 traitements «matières organiques " provoquent des accroissements de rendement en grain par rapport au témoin « $T$ » (8 répétitions); il n'y a pas de différence entre les amendements.

\section{Pomme de terre (tabl. 5)}

Les pommes de terre ont été cultivées pendant 8 ans (de 1970 à 1976 et en 1978). Chaque année, le poids de tubercules, le nombre de tubercules et le poids des fanes sont plus faibles dans les parcelles témoins " $T$ » que dans les parcelles recevant des amendements organiques. Ces différences sont significatives 7 années sur 8 dans le cas du poids de tubercules, 6 années sur 8 pour le nombre de tubercules et le poids de fanes. Il n'y a par contre jamais de différence significative entre les différents amendements organiques.

L'effet de la matière organique est plus important sur les parties aériennes que sur les tubercules. Les amendements accroissent légèrement le poids moyen des tubercules.

\section{Conclusion}

Dans nos conditions expérimentales, les amendements organiques provoquent des augmentations significatives de rendement par rapport au témoin pour 8 récoltes sur 12 (bien que le niveau moyen des rendements soit élevé :
88 q/ha de grain aux normes - à 15 p. 100 d'humidité pour le mais, $35 \mathrm{t} / \mathrm{ha}$ de tubercules pour la pomme de terre).

La différence d'effet des matières organiques sur le maïs et la pomme de terre peut avoir 2 origines :

- effet cumulatif et ne se manifestant qu'après 2 ou 3 années d'apports, quand les propriétés des sols ont été suffisamment modifiées ;

- moindre réaction du maïs à un apport humique.

\section{B. Effet des matières organiques sur les propriétés des sols}

Les résultats de cet essai confirment et complètent ceux que nous avions obtenus précédemment (DELAS, 1971).

\section{1. Évolution de la teneur en matière organique}

La teneur en matière organique a été mesurée par dosage de l'azote total et du carbone organique. Les teneurs initiales (1967) et finales (1978) en azote et carbone des 20 premiers $\mathrm{cm}$ sont indiquées au tableau 6 .

Le taux de matière organique des parcelles témoins « $T$ » qui n'ont reçu aucun amendement pendant toute la durée de l'essai diminue nettement de 1967 à 1978. Le rythme de disparition de la matière organique s'établit à 2,5 p. 100 par an quand il est mesuré par le carbone ou à 1,7 p. 100 par an quand il est mesuré par l'azote (soit 2 p. 100 en moyenne). Ces valeurs sont à rapprocher de celles que nous avions obtenues précédemment dans un sol identique : 1,9 p. 100 pour le carbone, 3,0 p. 100 pour l'azote (DELAS, 1971); les légères divergences s'expliquent par le fait que le $1^{\text {cr }}$ essai, contrairement au second, ne comportait ni culture ni apport d'azote minéral.

Les divers amendements contribuent à enrichir le sol en matière organique. Bien que les quantités de carbone apportées aient été les mêmes dans tous les traitements, en fin d'essai le stock de carbone et le rapport $\mathrm{C} / \mathrm{N}$ sont très variables. Nous avons pu calculer le rendement en carbone stable (fraction du carbone apporté présente dans le sol à la fin de l'essai) des divers amendements (tabl. 7).

En ce qui concerne l'azote, pour lequel les apports par les amendements et par la fumure minérale sont beaucoup plus variables, l'enfouissement des pailles de blé (traitement « $\mathrm{P} »)$ ou d'écorces de résineux (traitement « $\mathrm{E} »)$ suffit à peine à maintenir le taux initial.

\section{TABLEAU 4}

Influence des amendements organiques sur les rendements du mä̈s (moyenne des répétitions). Effect of organic materials on maize yields (mean of replicates).

\begin{tabular}{|c|c|c|c|c|c|c|c|}
\hline Annéc & $\begin{array}{c}\mathrm{T} \\
\text { Témoin }\end{array}$ & $\underset{\substack{\text { Marcs de } \\
\text { raisin }}}{R}$ & $\begin{array}{c}E \\
\text { Ecorces de } \\
\text { résineux }\end{array}$ & $\begin{array}{c}\mathbf{M} \\
\text { Tiges de } \\
\text { maïs }\end{array}$ & $\begin{array}{c}F \\
\text { Fumicr } \\
\text { de ferme }\end{array}$ & $\begin{array}{c}\text { P } \\
\text { Paille } \\
\text { de blé }\end{array}$ & $\begin{array}{l}\text { Signification } \\
\text { statistique }\end{array}$ \\
\hline \multicolumn{8}{|c|}{ 1. Rendement en grain (t/ha de matière sèche) } \\
\hline $\begin{array}{c}1967 \\
1968 \\
1969 \\
1977 \\
\text { Moyenne }\end{array}$ & $\begin{array}{l}5,34 \\
8,07 \\
8,41 \\
6,23 \\
7,01\end{array}$ & $\begin{array}{l}4,60 \\
8,85 \\
8,83 \\
7,99 \\
7,57\end{array}$ & $\begin{array}{l}4,82 \\
8,37 \\
8,16 \\
8,12 \\
7,37\end{array}$ & $\begin{array}{l}4,67 \\
8,28 \\
7,87 \\
7,84 \\
7,17\end{array}$ & $\begin{array}{l}6,72 \\
9,20 \\
8,79 \\
8,62 \\
8,33\end{array}$ & $\begin{array}{l}6,04 \\
8,95 \\
8,45 \\
7,95 \\
7,85\end{array}$ & $\begin{array}{l}\text { N.S. } \\
\text { N.S. } \\
\text { N.S. } \\
1 \%\end{array}$ \\
\hline \multicolumn{8}{|c|}{ II. Poids des tiges (t/ha de matière sèche) } \\
\hline $\begin{array}{c}1967 \\
1968 \\
1969 \\
1977 \\
\text { Moyenne }\end{array}$ & $\begin{array}{l}2,18 \\
3,33 \\
6,12 \\
5,11 \\
4,19\end{array}$ & $\begin{array}{l}1,70 \\
3,92 \\
2,44 \\
5,14 \\
3,30\end{array}$ & $\begin{array}{l}1,69 \\
2,77 \\
3,08 \\
7,34 \\
3,72\end{array}$ & $\begin{array}{l}1,49 \\
2,83 \\
2,61 \\
6,98 \\
3,48\end{array}$ & $\begin{array}{l}2,83 \\
3,10 \\
3,24 \\
6,63 \\
3,95\end{array}$ & $\begin{array}{l}2,01 \\
3,94 \\
2,91 \\
5,46 \\
3,58\end{array}$ & $\begin{array}{l}\text { N.S. } \\
\text { N.S. } \\
\text { N.S. } \\
5 \%\end{array}$ \\
\hline
\end{tabular}




\section{TABLEAU 5}

Influence des amendements organiques sur les rendements de la pomme de terre (moyenne des répétitions). Effect of organic materials on potato yields (mean of replicates).

\begin{tabular}{|c|c|c|c|c|c|c|c|}
\hline Année & $\begin{array}{c}\mathrm{T} \\
\text { Témoin }\end{array}$ & $\begin{array}{c}\mathbf{R} \\
\text { Marcs de } \\
\text { raisin }\end{array}$ & $\begin{array}{c}E \\
\text { Ecorces de } \\
\text { résineux }\end{array}$ & $\begin{array}{c}\mathbf{M} \\
\text { Tiges de } \\
\text { maïs }\end{array}$ & $\begin{array}{c}F \\
\text { Fumier } \\
\text { de ferme }\end{array}$ & $\begin{array}{c}\text { P } \\
\text { Paille } \\
\text { de blé }\end{array}$ & $\begin{array}{l}\text { Signification } \\
\text { statistique }\end{array}$ \\
\hline \multicolumn{8}{|c|}{ I. Poids frais des tubercules (t/ha) } \\
\hline $\begin{array}{l}1970 \\
1971 \\
1972 \\
1973 \\
1974 \\
1975 \\
1976 \\
1978 \\
\text { Moyenne }\end{array}$ & $\begin{array}{l}20,44 \\
33,44 \\
13,29 \\
39,19 \\
41,63 \\
26,01 \\
22,28 \\
17,22 \\
26,69\end{array}$ & $\begin{array}{l}30,41 \\
37,36 \\
11,26 \\
44,30 \\
55,69 \\
48,20 \\
32,06 \\
27,87 \\
35,89\end{array}$ & $\begin{array}{l}26,69 \\
40,11 \\
15,50 \\
45,98 \\
59,03 \\
45,63 \\
30,94 \\
24,13 \\
36,00\end{array}$ & $\begin{array}{l}29,50 \\
43,33 \\
20,78 \\
42,87 \\
60,80 \\
48,03 \\
34,69 \\
26,50 \\
38,31\end{array}$ & $\begin{array}{l}25,22 \\
39,50 \\
13,23 \\
40,01 \\
48,64 \\
45,40 \\
34,58 \\
25,92 \\
34,06\end{array}$ & $\begin{array}{l}26,83 \\
43,08 \\
17,58 \\
42,90 \\
50,63 \\
34,84 \\
27,61 \\
26,26 \\
33,72\end{array}$ & $\begin{array}{l}1 \% \\
1 \% \\
5 \% \\
5 \% \\
1 \% \\
1 \% \\
\text { N.S. } \\
1 \%\end{array}$ \\
\hline \multicolumn{8}{|c|}{ II. Nombre de tubercules } \\
\hline $\begin{array}{c}1970 \\
1971 \\
1972 \\
1973 \\
1974 \\
1975 \\
1976 \\
1978 \\
\text { Moyenne }\end{array}$ & $\begin{array}{l}282,25 \\
278,50 \\
179,25 \\
308,12 \\
249,37 \\
238,00 \\
229,37 \\
180,00 \\
243,11\end{array}$ & $\begin{array}{l}417,75 \\
344,75 \\
158,25 \\
397,25 \\
322,37 \\
358,25 \\
295,12 \\
215,25 \\
313,62\end{array}$ & $\begin{array}{l}356,75 \\
319,25 \\
188,62 \\
413,00 \\
324,62 \\
411,75 \\
317,87 \\
217,00 \\
318,61\end{array}$ & $\begin{array}{l}330,75 \\
347,00 \\
211,75 \\
368,62 \\
318,50 \\
338,50 \\
311,87 \\
208,50 \\
304,44\end{array}$ & $\begin{array}{l}321,25 \\
367,50 \\
146,62 \\
364,62 \\
289,75 \\
300,87 \\
311,00 \\
219,12 \\
290,99\end{array}$ & $\begin{array}{l}347,50 \\
319,75 \\
218,87 \\
349,87 \\
290,37 \\
259,37 \\
264,87 \\
214,37 \\
283,12\end{array}$ & $\begin{array}{c}1 \% \\
1 \% \\
\text { N.S. } \\
1 \% \\
1 \% \\
1 \% \\
\text { N.S. } \\
5 \%\end{array}$ \\
\hline \multicolumn{8}{|c|}{ III. Poids frais des fanes } \\
\hline $\begin{array}{l}1970 \\
1971 \\
1972 \\
1973 \\
1974 \\
1975 \\
1976 \\
1978 \\
\text { Moyenne }\end{array}$ & $\begin{array}{r}17,41 \\
9,24 \\
2,52 \\
10,23 \\
17,89 \\
12,03 \\
22,28 \\
4,73 \\
12,04\end{array}$ & $\begin{array}{r}27,83 \\
10,97 \\
3,41 \\
10,06 \\
21,41 \\
27,94 \\
32,06 \\
8,86 \\
17,82\end{array}$ & $\begin{array}{r}26,28 \\
13,28 \\
3,26 \\
12,70 \\
29,01 \\
31,96 \\
30,94 \\
7,74 \\
19,40\end{array}$ & $\begin{array}{r}26,91 \\
12,52 \\
4,23 \\
11,67 \\
28,11 \\
27,87 \\
34,69 \\
8,26 \\
19,28\end{array}$ & $\begin{array}{r}21,39 \\
12,72 \\
3,48 \\
7,79 \\
15,36 \\
20,63 \\
34,58 \\
7,36 \\
15,41\end{array}$ & $\begin{array}{r}23,41 \\
14,13 \\
3,68 \\
12,30 \\
23,64 \\
21,03 \\
27,61 \\
7,42 \\
16,65\end{array}$ & $\begin{array}{l}1 \% \\
5 \% \\
\text { N.S. } \\
1 \% \\
1 \% \\
1 \% \\
\text { N.S. } \\
1 \%\end{array}$ \\
\hline
\end{tabular}

TABLEAU 6

Evolution des teneurs en azote et en carbone dans les 20 premiers $\mathrm{cm}$ de sol de 1967 à 1978 (en \%o de terre fine) (moyenne des 8 répétitions). Variation in nitrogen and carbon content during the 1967 to 1978 period in top $20 \mathrm{~cm}$ of soil (\%o of fine earth) (mean of 8 replicates).

\begin{tabular}{|c|c|c|c|c|c|c|c|}
\hline \multirow{2}{*}{ Traitements } & \multicolumn{3}{|c|}{ Azote } & \multicolumn{3}{|c|}{ Carbone } & \multirow{2}{*}{$\begin{array}{l}\mathrm{C} / \mathrm{N} \\
1978\end{array}$} \\
\hline & 1967 & 1978 & $\begin{array}{c}\text { Signification } \\
\text { statistique }\end{array}$ & 1967 & 1978 & $\begin{array}{c}\text { Signification } \\
\text { statistique }\end{array}$ & \\
\hline T. Témoin & 0,65 & 0,53 & $1 \%$ & 7,5 & 5,6 & $1 \%$ & 10,56 \\
\hline R. Marcs de raisin & 0,64 & 1,09 & $1 \%$ & 7,4 & 11,6 & $1 \%$ & 10,64 \\
\hline M. Tiges de maïs & 0,58 & 0,68 & $1 \%$ & 6,9 & 9,1 & $1 \%$ & 13,38 \\
\hline F. Fumier de ferme & 0,68 & 0,96 & $1 \%$ & 7,8 & 10,8 & $1 \%$ & 11,25 \\
\hline P. Paille de blé & 0,65 & 0,65 & N.S. & 7,4 & 7,9 & N.S. & 12,15 \\
\hline
\end{tabular}

2. Capacité d'échange de cations, C.E.C. (tabl. 8)

La C.E.C. augmente significativement entre 1967 et 1978 dans les 2 traitements pour lesquels les teneurs en azote total et carbone organique ont été le plus fortement accrues (marcs de raisin « $R$ » et fumier « $F »)$. Dans les parcelles témoins « $\mathrm{T}$ », et comme conséquence de leur appauvrissement en matière organique, la capacité d'échange diminue de 1967 à 1978.
La relation entre C.E.C. et teneur en azote et carbone que nous avions établie précédemment (DELAS, 1971) est vérifiée dans cet essai.

3. $\mathrm{P}_{2} \mathrm{O}_{5}$ assimilable; $\mathrm{K}, \mathrm{Ca}$ et $\mathrm{Mg}$ échangeables (tabl. 8)

Les teneurs en $\mathrm{P}_{2} \mathrm{O}_{5}$ assimilable, $\mathrm{K}$ et $\mathrm{Mg}$ échangeables augmentent légèrement entre 1967 et 1978 mais ne varient pas beaucoup d'un traitement à l'autre: le système des 


\section{TABLEAU 7}

Rendement en carbone stable des matières organiques apportées. Stable carbon yield from organic materials supplied (\% of added organic matter).

\begin{tabular}{|c|c|}
\hline R. Marcs de raisin & 0,37 \\
\hline E. Ecorces de résineux & 0,31 \\
\hline 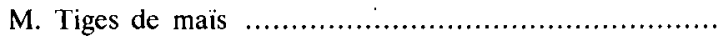 & 0,20 \\
\hline 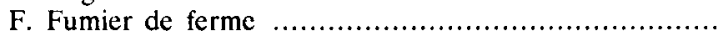 & 0,32 \\
\hline 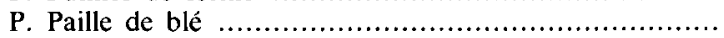 & 0,14 \\
\hline
\end{tabular}

Un hectare de terre fine pèse sur $20 \mathrm{~cm}: 2000$ tonnes compensations adopté permet donc, pour ces 3 éléments, de maintenir ou d'augmenter légèrement la fertilité du sol. Par contre, dans le même temps, le taux de calcium initialement élevé en raison d'épandages antérieurs de scories - diminue considérablement malgré les apports effectués, sous forme de sulfate, au titre des compensations. Cette diminution doit être attribuée au lessivage du calcium par les eaux de pluie et d'irrigation. Ce lessivage est en effet important dans les sols sableux: JUSTE et al. (1982) ont trouvé des pertes de calcium de l'ordre de $200 \mathrm{~kg} / \mathrm{ha} / \mathrm{an}$ dans un sol sableux des Landes de Gascogne (podzol riche en matière organique) soumis à une monoculture irriguée de maïs. Le lessivage du calcium est cependant réduit dans les traitements « $R$ » et « $F$ » dont la C.E.C. est la plus élevée.

TABLEAU 8

Evolution des teneurs en $\mathrm{P}_{2} \mathrm{O}_{5}$ assimilable, de la C.E.C. et des teneurs en $\mathrm{K}$, Ca et $\mathrm{Mg}$ échangeables des 20 premiers $\mathrm{cm}$ de sol de 1967 à 1978 (moyenne des 8 répétitions).

Variation in soil available $\mathrm{P}_{2} \mathrm{O}_{5}$, cation exchange capacity, exchangeable $\mathrm{K}, \mathrm{Ca}$ and $\mathrm{Mg}$ during the 1967 to 1978 period in top $20 \mathrm{~cm}$ of soil (mean of 8 replicates).

\begin{tabular}{|c|c|c|c|c|c|c|c|c|c|c|c|c|c|c|c|}
\hline \multirow{2}{*}{ Traitements } & \multicolumn{3}{|c|}{$\mathrm{P}_{2} \mathrm{O}_{5} \%$} & \multicolumn{3}{|c|}{ C.E.C. mé $/ 100 \mathrm{~g}$} & \multicolumn{3}{|c|}{$K \%$} & \multicolumn{3}{|c|}{$\mathrm{Ca} \%$} & \multicolumn{3}{|c|}{$\mathrm{Mg} \%$} \\
\hline & 1967 & 1978 & $\begin{array}{l}\text { Signif. } \\
\text { statis. }\end{array}$ & 1967 & 1978 & $\begin{array}{l}\text { Signif. } \\
\text { statis. }\end{array}$ & 1967 & 1978 & $\begin{array}{l}\text { Signif. } \\
\text { statis. }\end{array}$ & 1967 & 1978 & $\begin{array}{l}\text { Signif. } \\
\text { statis. }\end{array}$ & 1967 & 1978 & $\begin{array}{l}\text { Signif. } \\
\text { statis. }\end{array}$ \\
\hline T. Témoin & 0,293 & 0,417 & $1 \%$ & 4,8 & 4,3 & $1 \%$ & 0,070 & 0,086 & N.S. & 1,275 & 0,447 & $5 \%$ & 0,025 & 0,031 & N.S. \\
\hline R. Marcs de raisin & 0,288 & 0,368 & $1 \%$ & 5,0 & 6,3 & $1 \%$ & 0,075 & 0,070 & N.S. & 1,146 & 0,905 & $1 \%$ & 0,036 & 0,044 & N.S. \\
\hline E. Ecorces de résineux & 0,285 & 0,387 & $1 \%$ & 5,0 & 5,4 & N.S. & 0,061 & 0,098 & $1 \%$ & 1,335 & 0,594 & $1 \%$ & 0,024 & 0,036 & N.S. \\
\hline M. Tiges de maïs & 0,258 & 0,396 & $1 \%$ & 4,9 & 4,9 & N.S. & 0,054 & 0,093 & $1 \%$ & 1,116 & 0,579 & $1 \%$ & 0,033 & 0,039 & N.S. \\
\hline F. Fumier de ferme & 0,300 & 0,365 & $1 \%$ & 5,1 & 6,0 & $1 \%$ & 0,086 & 0,115 & $5 \%$ & 1,134 & 0,975 & $5 \%$ & 0,037 & 0,050 & N.S. \\
\hline P. Paille de blé & 0,287 & 0,400 & $1 \%$ & 5,0 & 5,1 & N.S. & 0,072 & 0,090 & $5 \%$ & 1,075 & 0,532 & $1 \%$ & 0,034 & 0,035 & N.S. \\
\hline
\end{tabular}

\section{Evolution du pH (tabl. 9)}

Entre 1967 et 1978 , le $\mathrm{pH}$ du sol de tous les traitements diminue très nettement (de plus de 2 unités en général). Les marcs de raisin $(« \mathrm{R} »)$, pourtant réputés acidifiants, ne se distinguent pas dans cet essai des autres traitements. L'acidification générale constatée doit être attribuée à l'effet des engrais azotés minéraux et à la facilité d'élimination du calcium dans ce type de sol.

\section{Capacité de rétention pour l'eau (tabl. 9)}

Les apports d'amendements provoquent une augmentation de la capacité initiale de rétention pour l'eau allant de 10 à 30 p. 100 .

\section{TABLEAU 9}

Evolution du pH et de la capacité de rétention pour l'eau dans les 20 premiers cm de sol de 1967 à 1978 (moyenne des 8 répétitions). Variation in soil $\mathrm{pH}$ and water-holding capacity during the 1967 to 1978 period in top $20 \mathrm{~cm}$ of soil (mean of 8 replicates).

\begin{tabular}{|c|c|c|c|c|c|c|c|c|c|}
\hline & \multicolumn{3}{|c|}{ pH-eau } & \multicolumn{3}{|c|}{$\mathrm{pH}-\mathrm{KCl}$} & \multicolumn{3}{|c|}{$\begin{array}{c}\text { Capacité de rétention } \\
\text { pour l'eau } \\
(\% \text { de terre fine })\end{array}$} \\
\hline & 1967 & 1978 & $\begin{array}{l}\text { Signif. } \\
\text { statis. }\end{array}$ & 1967 & 1978 & $\begin{array}{l}\text { Signif. } \\
\text { statis. }\end{array}$ & 1967 & 1978 & $\begin{array}{l}\text { Signif. } \\
\text { statis. }\end{array}$ \\
\hline R. Marcs de raisin & 7,0 & 5,3 & $1 \%$ & 6,5 & 4,9 & $1 \%$ & 8,0 & 9,7 & $1 \%$ \\
\hline E. Ecorces de résineux & 7,2 & 4,6 & $1 \%$ & 6,6 & 4,3 & $1 \%$ & 7.7 & 8,8 & $1 \%$ \\
\hline M. Tiges de maïs & 7,2 & 4,8 & $1 \%$ & 6,6 & 4,4 & $1 \%$ & 7,4 & 8,8 & $1 \%$ \\
\hline F. Fumier de ferme & 7,0 & 5,7 & $1 \%$ & 6,3 & 5,3 & $1 \%$ & 8,2 & 10,3 & $1 \%$ \\
\hline P. Paille de blé & 7,0 & 4,9 & $1 \%$ & 6,4 & 4,3 & $1 \%$ & 8,2 & 9,4 & $1 \%$ \\
\hline
\end{tabular}




\section{DISCUSSION}

Dans cet essai, où l'égalité des apports minéraux entre parcelles témoins et parcelles recevant diverses matières organiques est réalisée, les amendements entraînent des augmentations de production importantes; les engrais minéraux seuls ne semblent pas permettre d'atteindre le rendement le plus élevé. Ces résultats diffèrent de ceux de Muller (1965) qui, dans un essai analogue sous climat méditerranéen, n'a pas observé d'effet spécifique des amendements organiques. L'origine de ces différences est à rechercher dans la nature des sols des 2 essais: le sol sableux de notre dispositif, très pauvre en matière organique, est plus favorable à l'expression d'un effet de la matière organique que le sol du dispositif de MuLLER, calcaire et bien pourvu en colloïdes minéraux et organiques.

Avant de passer en revue les hypothèses permettant d'expliquer l'effet des amendements sur les rendements du maïs et de la pomme de terre, nous voudrions revenir sur le protocole expérimental adopté: nous avons apporté la même quantité de carbone avec les divers traitements et effectué, grâce à des compléments minéraux, une double compensation afin de réaliser l'égalité des apports entre traitements et de compenser les exportations par les récoltes. Ces précautions, pour indispensables qu'elles soient, ne suffisent pas à assurer l'uniformité de la fertilité minérale du sol des parcelles. Les compensations sont en effet limitées à 5 éléments ( $\mathrm{N}, \mathrm{P}, \mathrm{K}, \mathrm{Ca}$ et $\mathrm{Mg}$ ) ; aucun chaulage n'a été réalisé ; mais surtout les parcelles témoins « $T$ » ont en définitive reçu une fertilisation différente de celle des autres traitements : forme exclusivement minérale (organominérale pour les autres traitements) susceptible d'entraîner des phénomènes de toxicité, doses plus faibles du fait de rendements inférieurs susceptibles, au contraire, d'entraîner des phénomènes de carence. En fait, l'examen du tableau 3 permet de constater que les parcelles témoins « $T$ » n'ont pas été pénalisées : elles ont reçu une fumure minérale tout à fait comparable à celle des parcelles donnant des rendements nettement plus élevés (tiges de maïs « $\mathrm{M}$ », écorces de résineux « $\mathrm{E}$ », pailles de blé $~ « \mathrm{P} »)$ et très supérieure aux besoins des 2 cultures irriguées ; dans ce traitement « $T$ », les apports moyens d'azote, d'anhydride phosphorique et d'oxyde de potassium ont été (en $\mathrm{kg} / \mathrm{ha} / \mathrm{an}$ ) de : $350 \mathrm{~N}-140 \mathrm{P}_{2} \mathrm{O}_{5}-410 \mathrm{~K}_{2} \mathrm{O}$ pour le maïs, et de $280 \mathrm{~N}-110 \mathrm{P}_{2} \mathrm{O}_{5}-360 \mathrm{~K}_{2} \mathrm{O}$ pour la pomme de terre.

Les différences de rendements observées dans nos conditions expérimentales ne sont donc pas la conséquence du système de la double compensation mais ont bien été induites par les traitements.

Il nous faut maintenant essayer de relier ces différences de rendement aux modifications des propriétés des sols par les traitements.

Les teneurs du sol en $\mathrm{P}_{2} \mathrm{O}_{5}, \mathrm{~K}$ et $\mathrm{Mg}$ ne semblent jouer aucun rôle. Les teneurs initiales étaient élevées dans le cas de $\mathrm{P}_{2} \mathrm{O}_{5}$, moyennes (pour un sol sableux) dans le cas de $\mathrm{K}$ et $\mathrm{Mg}$. Elles ont été légèrement augmentées entre 1967 et 1978 mais sont restées du même ordre de grandeur pour les différents traitements; les parcelles témoins « $T$ » en particulier ne sont pas plus pauvres que les autres. Cette relative constance est la conséquence du système des compensations adopté.

De même, l'acidification générale du sol du dispositif (explicable par les forts apports d'azote minéral et l'absence du chaulage) n'a pas joué puisqu'elle est aussi importante dans les parcelles témoins « $T$ » que dans les parcelles les plus productives. En outre, le niveau moyen des rendements des 2 cultures, bien adaptées aux sols acides, est élevé.
Parmi les paramètres mesurés, c'est la teneur en matière organique qui a le plus varié sous l'influence des traitements. Mais il n'est pas possible d'en déduire que les variations de cette teneur sont responsables des différences de rendement observées : bien qu'ils aient enrichi le sol en matière organique de manière très différente, les amendements ont tous eu le même effet sur les rendements. Nous pouvons simplement observer que 2 facteurs ont été accrus par les apports d'amendements : la capacité de rétention pour l'eau (dans tous les cas) et la capacité d'échange de cations (accroissement significatif dans 2 traitements). Malgré l'irrigation et une fertilisation intensive, la matière organique aurait pu assurer une meilleure alimentation hydrique et minérale des cultures dans ce sol très filtrant.

Un effet direct des amendements sur les mécanismes d'élaboration du rendement reste du domaine des hypothèses; nous remarquerons cependant que, dans le cas de la pomme de terre, les amendements modifient le nombre de tubercules et non leur poids moyen.

En définitive, les amendements ont tous exercé un effet bénéfique sur les rendements; la nature du dispositif expérimental et le protocole adopté ne permettent pas d'expliquer l'origine de cet effet qui ne peut cependant pas être attribué à la richesse en éléments minéraux de ces amendements compte tenu des modalités de compensations mises en œuvre. Certaines modifications des caractéristiques du sol ont probablement joué un rôle (capacité d'échangę de cations, capacité de rétention en eau), mais on ne doit pas exclure l'intervention possible d'autres facteurs (par exemple, augmentation de la rhizogenèse chez la pomme de terre).

Par ailleurs, l'essai nous a permis de recueillir des informations concernant l'évolution des sols. En l'absence d'apports organiques (traitement témoin « $T »$ ), la vitesse de disparition des composés humiques s'établit à environ 2 p. 100 par an. Ce résultat est en accord avec les données que nous avons recueillies précédemment dans un sol sableux analogue (DELAS, 1971) mais aussi avec des données récentes obtenues dans des conditions pédoclimatiques très différentes (DELPHIN \& CONESA, 1979; LUBET \& JUSTE, 1979).

Nous avons également calculé le rendement en carbone stable des matières organiques utilisées. Les résultats obtenus doivent être interprétés avec précaution parce que le prélèvement de sol a été fait un an seulement après le dernier apport et aussi parce que les conditions d'incorporation au sol des amendements constitués de résidus de culture (paille de blé, tiges de maiis) ont été très différentes dans notre expérimentation de ce qu'elles sont dans la pratique agricole. Ce rendement en carbone stable est probablement différent du rendement en humus, ou coefficient isohumique, dont la détermination nécessite un isolement de la matière organique stable difficile à réaliser en sol sableux (DELAS, 1971). Il faut cependant remarquer que les rendements en carbone stable que nous avons trouvés pour la paille de blé, les tiges de maïs ou le fumier de ferme sont du même ordre de grandeur que les coefficients isohumiques cités par HÉnIN (1945) et par DELPHIN \& CONESA (1979) ; par contre, LUBET \& JUSTE (1979) ont trouvé, pour les tiges de maïs, un coefficient isohumique beaucoup plus faible (moins de 10 p. 100 au lieu de 20 p. 100) qui peut être attribué à des conditions de minéralisation plus intenses dans un sol à texture très différente.

Notre essai a permis enfin de situer les amendements dont le rendement en carbone stable est mal connu (marcs de raisin, écơrces de résineux) par rapport aux amendements plus classiquement étudiés: le rendement des marcs de 
raisin (37 p. 100) et des écorces de résineux (31 p. 100) est voisin de celui du fumier de ferme $(32$ p. 100$)$ et sensiblement plus élevé que celui des tiges de maïs $(20 \mathrm{p} .100)$ et surtout des pailles de blé (14 p. 100).

\section{CONCLUSION}

Dans un sol sableux, pauvre en matière organique, nous avons obtenu, en plein champ, grâce à des apports d'amendements organiques d'origines variées mais contenant la même quantité de carbone, des accroissements importants du rendement de la pomme de terre et du maïs irrigués (par rapport au témoin). L'origine de ces différences de rendement observées malgré l'égalité des apports minéraux entre traitements (et un niveau de fertilisation minérale élevé) n'a pas été clairement établie ; l'effet sur la production des différents amendements a été identique.

Ces résultats peuvent être difficilement transposés à d'autres milieux et à d'autres cultures: ils concernent des sols très particuliers, auxquels ont été incorporées régulièrement et avec soin des quantités importantes d'amendements; en outre, l'effet de la matière organique s'est essenticllement manifesté sur la pomme de terre.

Mais les sols sableux représentent des surfaces non négligeables en France; en Aquitaine, ils portent des cultures d'un grand intérêt économique (vigne dans les sols sableux de graves du Bordelais, mais dans les sols sableux des Landes). Dans ces sols dépourvus de colloïdes minéraux, la matière organique est un élément essentiel de la fertilité et, de ce fait, le problème des restitutions organiques y revêt une particulière importance. C'est ainsi, par exemple, que nos résultats amènent à déconseiller l'utilisation à des fins énergétiques des résidus de récolte du maïs cultivé en sol sableux pauvre en matière organique: le niveau satisfaisant du rendement dans le traitement tiges de maïs « $M$ " est dû à l'enfouissement annuel d'une quantité de tiges correspondant en fait aux résidus de la culture ( $6 \mathrm{t} /$ ha de matière sèche) .

Cet essai a également permis de préciser l'effet de divers amendements sur les propriétés du sol. En l'absence de restitutions organiques, la vitesse de disparition de la matière organique s'établit en sol sableux à environ 2 p. 100 par an. Les données recueillies soulignent l'intérêt de sousproduits trop souvent négligés, comme les marcs de raisin et les écorces de résineux, dont le rendement en carbone stable est assez élevé.

Reçu le 4 février 1982. Accepté le 12 août 1982.

\section{RÉFÉRENCES BIBLIOGRAPHIQUES}

Anne P., 1945. Sur le dosage rapide du carbone organique des sols. Ann. agron., 15, 161-172.

Delas J., 1971. Evolution des propriétés d'un sol sablcux sous l'influence d'apports massifs et répétés de matières organiques de différentes origines. Ann. agron., 22, 585-610.

Delas J., Molot C., 1968. Evolution de la teneur en matière organique des sols du vignoble bordelais. C.R. Acad. Agric. Fr., 54, 929-933.

Delas J., Juste C., Goulas J.-P., 1973. Matières organiques et fertilité des sols. Contribution à l'étude des effets de la matière organique sur les rendements et la qualité des récoltes ainsi que sur l'évolution du milieu. Bull. Tech. Inf., Min. Agric., 285, 841-855. Delphin J.-E., Conesa A.-P., 1979. Evolution de la matière organique du sol d'un cssai rotation, irrigation, restitution des pailles, dans la plaine de la Hardt. Ann. agron., 30, 167-190.
Hénin S., Dupuis M., 1945. Essai de bilan de la matière organique du sol. Ann. agron., 15, 17-29.

Juste C., Tauzin J., Dureau P., Courpron C., 1982. Exportation des éléments fertilisants par lessivage en sol sableux des Landes de Gascogne. Résultats de 8 années d'observations en cases lysimétriques. Agronomie, 2, 91-98.

Lubet E., Juste C., 1979. Effets de l'introduction d'une prairie temporaire, d'un engrais vert et de l'exportation des résidus de récolte sur les monocultures de mais implantées dans les sols sablolimoneux du Sud des Landes. C.R. Acad. Agric. Fr., 65, 295-309. Muller J., 1965. Observations sur les effets à long terme des fumures organiques et minérales sous climat méditerranéen. I. Action sur les rendements. Ann. agron., 16, 301-321. 